\title{
Pulmonary Mucormycosis in an Immunocompetent Patient: A Rare Life-threatening Case
}

\author{
Cenk Balta \\ Department of Thoracic Surgery, Faculty of Medicine, Balikesir University, Balikesir, Turkey
}

\begin{abstract}
Pulmonary mucormycosis is a rare but life-threatening, fungal infection that usually occurs in patients with immunocompromised conditions, such as diabetes mellitus and hematologic malignancies. Anti-fungals and/or surgery are treatment modalities for this disease. Isolated pulmonary mucormycosis in immunocompetent patient is extremely rare and a mortal fungal infection. Late diagnosis may cause increased morbidity and mortality. We report herein a case of an immunocompetent 37-year female, who presented with a massive hemoptysis and was treated with urgent right lower lobectomy on a suspicion of fungus ball on tomography scan. The specimen was reported as pulmonary mucormycosis on histopathological examination.
\end{abstract}

Key Words: Mucormycosis, Hemoptysis, Cavitary lesion, Immunocompetent.

How to cite this article: Balta C. Pulmonary Mucormycosis in an Immunocompetent Patient: A Rare Life-threatening Case. J Coll Physicians Surg Pak 2022; 32(03):386-388.

\section{INTRODUCTION}

Mucormycosis is an opportunistic, life-threatening, fungal infection that is usually seen in patients with immune system defects. It is associated with high mortality and morbidity. Mucormycosis is the third most common invasive fungal infection following the candidiasis and aspergillosis. ${ }^{1}$ It was firstly described in 1876 by Furbringer. The most common fungus types causing mucormycosis are: rhizopus, lichtheimia, cunninghamella and mucor. ${ }^{2,3}$

The optimal temperature for aerobic growth is 28 to $30^{\circ} \mathrm{C}$. Mucormycosis typically begins by attacking the respiratory tract as an airborne infection. It spreads to entire body by invading blood vessels in paranasal sinuses. ${ }^{3}$

There are six clinical forms of mucormycosis: rhinocerebral, pulmonary, cutaneous, gastrointestinal, disseminated, and uncommon forms such as osteomyelitis, peritonitis, and endocarditis. $^{3}$

Predisposing factors for mucormycosis are uncontrolled diabetesmellitus, diabeticketoacidosis, chemotherapy, hematological malignancies, immunosuppressive therapy, neutropenia, broad-spectrum antibiotic therapy, renal failure, solid organ transplantation, etc. ${ }^{1-3}$ Isolated pulmonary mucormycosis has been reported to be extremely rare in normal hosts. The incidence is reported as $6.25 \%$ in normal hosts. ${ }^{2,4}$

Correspondence to: Dr. Cenk Balta, Department of Thoracic Surgery, Faculty of Medicine, Balikesir University, Balikesir, Turkey

E-mail:drcenkbalta@gmail.com

Received: December 19, 2019; Revised: January 31, 2020;

Accepted: February 07, 2020

DOI: https://doi.org/10.29271/jcpsp.2022.03.386

\section{CASE REPORT}

A 37-year female presented to this hospital with a complaint of massive hemoptysis. There was no history of chronic illness. She had hemoptysis two months ago. Opacity in the right lower lobe was detected on the chest X-ray and oral levofloxacin $(400 \mathrm{mg}$ oncea day) was prescribed fortwo weeks.

On physical examination, blood pressure was $130 / 80 \mathrm{mmHg}$, heart rate was 100 beats per minute and respiratory rate, 22 breaths perminute. Cavernous bronchial sounds were audible at the right lung base posteriorly on the auscultation. The abdomen was soft without rebound tenderness. Electrocardiographic examination was normal.

The laboratory values were as follows: arterial blood $\mathrm{pH}: 7.42$ (normal: 7.35-7.45), partial $\mathrm{O}_{2}$ pressure $\left(\mathrm{PO}_{2}\right): 75 \mathrm{mmHg}$ (normal: $80-100 \mathrm{mmHg}$ ), partial $\mathrm{CO}_{2}$ pressure $\left(\mathrm{PCO}_{2}\right): 45 \mathrm{mmHg}$ (normal: $35-45 \mathrm{mmHg}$ ), white blood cell (WBC) count: $9.2 \times 10^{3} / \mu \mathrm{L}$ (normal: $4,5-11 \times 10^{3} / \mu \mathrm{L}$ ), hemoglobin $(\mathrm{Hb}): 11.3 \mathrm{~g} / \mathrm{dL}$ (normal: 13,1-17,2 g/dL), serum C-reactive protein (CRP): $57 \mathrm{mg} / \mathrm{L}$ (normal:0-5 mg/L), erythrocyte sedimentation rate (ESR): $52 \mathrm{~mm} / \mathrm{h}$ (normal: 0-15 $\mathrm{mm} / 1^{\text {st }} \mathrm{hr}$ ). Liver and renal function tests were normal.

Chest X-ray showed an oval-shaped lesion in the right lower lobe (Figure 1a) and thorax computed tomography (CT) (Figures $1 \mathrm{~b}$, c) confirmed thick-walled cavitations with air-fluid level in the same location. Differential diagnosis included fungal ball.

Flexible fiberoptic bronchoscopy was performed. The source of hemoptysis was located as right lower lobe bronchus; and attempt was made to stop the bleeding with cold saline and adrenalin solutions. A decision to undertake right lower lobectomy of the lung was made when hemoptysis recurred after 8 hours. Videothoracoscopy-assisted right lower lobectomy was 
performed and no further bleeding occurred after the operation. Tranexamic acid ( $500 \mathrm{mg}$ tablet, $2 \times 1$ ) was given postoperatively and the patient was discharged on the $5^{\text {th }}$ day of hospitalisation.

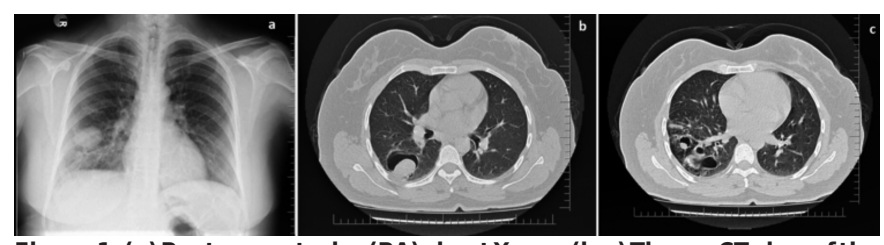

Figure 1: (a) Postero-anterior (PA) chestX-ray; $(b, c)$ Thorax CT view of the cavitary lesions.

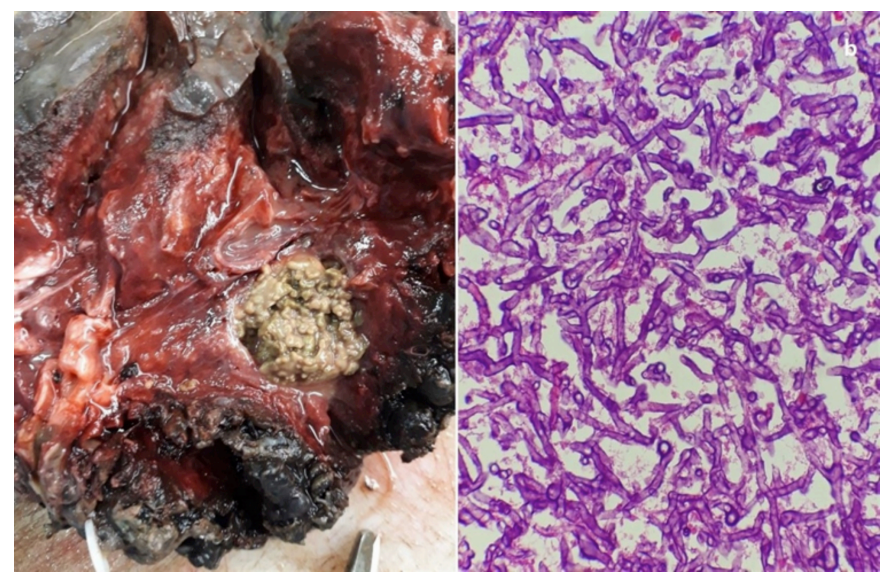

Figure 2: (a) Well-circumscribed, friable, grumous, gray color mass in the lung on gross examination; (b) Microscopy shows masses of non-septate, irregular, branching hyphae consistent with mucormycosis (H\&E, X400).

Pathologically, a gray-yellow colour fungus ball was noted on macroscopic examination (Figure 2a). Microscopic examination of the specimen showed thick-walled, non-septate, broad, rightangle branching hyphae (Figure $2 \mathrm{~b}$ ) consistent with pulmonary mucormycosis.

As there is a relation between mucormycosis and immune system defects, the patient was investigated for diabetes, malignancies and immune sufficiency. But, no related comorbidities were detected. The patient was referred to the infectious disease department of this hospital faculty; and it was decided that there is no need for further antifungal therapies. No recurrences were detected in the 14 months of follow-up.

Written informed consent was obtained from the patient for the publication of the study.

\section{DISCUSSION}

Mucormycosis is a life-threatening fungal disease that is caused by mucormycetes; and it has commonly been seen in immunocompromised patients. ${ }^{1}$ Pulmonary mucormycosis has a rapid fatal clinical progression; and prognosis is dependent on early diagnosis and aggressive treatment. The infection has a high mortality rate (50-70\%); in recent studies, surgical resection is recommended, if hemoptysis is massive. ${ }^{5}$

Clinical manifestations of the disease are categorised into six groups: rhinocerebral, cutaneous, pulmonary, gastrointestinal, central nervous system, and miscellaneous (bones, breasts, mediastinum, kidney etc.). ${ }^{3}$ Rhinocerebral mucormycosis is the most common form seen. The lungs are the most commonly affected sites in patients with the hematological diseases (64\%); and acute myeloid leukemia is the most common hematological malignancy. ${ }^{6}$ In addition, pulmonary mucormycosis is usually related with neutropenia and graft-versus-host disease. ${ }^{8}$ Isolated invasive pulmonary mucormycosis in normal hosts, as in our case, is extremely rare.

Symptoms of pulmonary mucormycosis are non-specific such as fever, cough, dyspnea, and chest pain. Prolonged fever is mostly seen in many patients with this infection, although it might be symptomless. ${ }^{7}$ Hemoptysis is possible due to erosion of the pulmonary artery into the tracheobronchial tree.

Diagnosis of pulmonary mucormycosis is challenging owing to its rarity. On radiological examination, pulmonary findings may include consolidation, infiltrations, air-crescent sign, halo sign (or reverse halo sign), solitary nodules, pleural effusions, and cavities. Lesions of the lung are commonly right-sided and involvement is commonly in upper lobes. There is bacterial pneumonia in $30 \%$ of pulmonary mucormycosis cases; and it may bea predictive factor for late diagnosis. ${ }^{8}$ Cavitation is observed in $40 \%$ of cases and air-crescent sign on $\mathrm{CT}$ is the precursor finding of fatal hemoptysis. ${ }^{9}$ The gold standard diagnostic method is cultureand histopathological examination is usually complementary. Demonstration of broad, non-septate, ribbon-like hyphae with right-angle branching is obligatory to diagnose mucormycosis. In aspergillosis, the hyphae are thinner, more organised, septate and show acute angle branching. ${ }^{5}$ In this case, there had been an emergency resection due to hemoptysis and a preoperative sample to microbiology department for culture cannot be sent because of urgentsurgery.

Clinical and radiological manifestations of aspergillosis and mucormycosis are quite similar. It is important to differentiate among these as appropriate treatments differs in both. In the medical treatment of mucormycosis, high-dose liposomal amphotericin B or intravenous isavuconazole and intravenous or delayed release tablet posaconazole is recommended. ${ }^{7}$ But these drugs are more effective, if surgery is included in the treatment. ${ }^{4,7}$ Voriconazole is not effective for this disease. ${ }^{10}$ Surgical resection and medical drug combination have lower mortality rates and provide better survival outcomes. The important part of treatment is not to delay the diagnosis and treatment. Survival is no longer than two weeks due to fungal sepsis, respiratory failure, and massive hemoptysis in untreated cases. In a study of 929 cases of mucormycosis, $8 \%$ cases $(n=176)$ were immunocompetent patients. Ten percent of cases had surgery alone and survival rate was $57 \%{ }^{2}$

In conclusion, isolated pulmonary mucormycosis is a rare fungal infection in immunocompetent patients and might be mortal, if the diagnosis is delayed. The clinical and radiologic manifestations can be non-specific. Thus, if the disease presents with hemoptysis, the clinicians should consider pulmonary mucormycosis in the differential diagnosis; and treatment modality should include early surgery and medical treatment combination. 


\section{PATIENT'S CONSENT:}

Informed consent was obtained from the patient to publish the data concerning this case.

\section{CONFLICT OF INTEREST:}

There are no conflicts of interest regarding employment, consultancies, stock ownership, honoraria, paid expert testimony, patentapplications/registrations, and grants or otherfunding.

\section{AUTHOR'S CONTRIBUTION:}

CB: Design, literature search, discussion and drafting.

\section{REFERENCES}

1. Kurosawa M, Yonezumi M, Hashino S, Tanaka J, Nishio M, Kaneda $M$, et al. Epidemiology and treatment outcome of invasive fungal infections in patients with hematological malignancies. Int J Hematol 2012; 96(6): 748-57. doi: 10. 1007/s12185-012-1210-y.

2. Roden MM, Zaoutis TE, Buchanan WL, Knudsen TA, Sarkisova TA, Schaufele RL, et al. Epidemiology and outcome of zygomycosis: A review of 929 reported cases. Clin Infect Dis 2005; 41(5):634-53. doi: 10.1086/432579.

3. Petrikkos G, Skiada A, Lortholary O, Roilides E, Walsh TJ, Kontoyiannis DP. Epidemiology and clinical manifestations of mucormycosis. Clin Infect Dis 2012; 54 (Suppl 1):S23-34. doi: 10.1093/cid/cir866.

4. Von Scheven R, Lebiedz P, Spieker T, Uekoetter A, Berdel WE, Kessler T. Fulminant invasive pulmonary mucormycosis with rhizopus oryzae in a patient with severe aplastic anaemia and common variable immunodeficiency. Mycoses 2012; 55(2):e32-5. doi: 10.1111/j.1439-0507. 2011.02119.x.

5. Ibrahim AS, Spellberg B, Walsh TJ, Kontoyiannis DP. Pathogenesis of mucormycosis. Clin Infect Dis 2012; 54 (Suppl 1):S16-22. doi.org/10.1093/cid/cir865.

6. Pagano L, Offidani M, Fianchi L, Nosari A, Candoni A, Picardi $M$, et al. Mucormycosis in hematologic patients. Haematologica 2004; 89(2): 207-14. doi.org/10.1093/ $\mathrm{cid} / \mathrm{cir} 865$.

7. Cornely $O A$, Alastruey Izquierdo A, Arenz D, Chen SCA, Dannaoui E, Hochhegger B, et al. Mucormycosis ECMM MSG global guideline writing group. Global guideline for the diagnosis and management of mucormycosis: An initiative of the European confederation of medical mycology in cooperation with the mycoses study group education and research consortium. Lancet Infect Dis 2019; 19(12):e405e21. doi: 10.1016/S1473-3099(19)30312-3.

8. Pavie J, Lafaurie M, Lacroix C, Marie Zagdanski A, Debrosse $D$, Socié $G$, et al. Successful treatment of pulmonary mucormycosis in an allogenic bonemarrow transplant recipient with combined medical and surgical therapy. Scand J Infect Dis 2004; 36(10):7679-9. doi: 10.1080/ 00365540410021081.

9. Fanci R, Pecile P, Di Lollo S, Dini C, Bosi A. Pulmonary mucormycosis with cervical lymph node involvement in a patient with acute myeloid leukaemia: A case report. Mycoses 2008; 51(4): 3546. doi: 10.1111/j.1439-0507. 2008.01494.x.

10. HuaPing Z, Jian L, JingBin $H$, Jie G, Guo-Xin M, Yan-Hong J, et al. Surgical resection and liposomal amphotericin $B$ to treat cavitary pulmonary zygomycosis in a patient with diabetes. Respir Care 2011; 56(11):18379. doi: 10.4187/respcare. 01119. 